\title{
BOILING VISUALIZATION ON VERTICAL FINS WITH TUNNEL-PORE STRUCTURES
}

\author{
Robert Pastuszko, Robert Kaniowski \\ Kielce University of Technology, Chair of Mechanics, \\ al. Tysiąclecia P.P.7, PL-25-314 Kielce, Poland \\ e-mail: tmprp@tu.kielce.pl
}

\begin{abstract}
The paper presents experimental studies of nucleate boiling heat transfer from a system of connected horizontal and vertical subsurface tunnels. The experiments were carried out for water at atmospheric pressure. The tunnel external covers were manufactured out of perforated copper foil (holes diameter $0.3 \mathrm{~mm}$ ), sintered with the mini-fins, formed on the vertical side of the $10 \mathrm{~mm}$ high rectangular fins and horizontal inter-fin surface. The image acquisition speed was 493 fps (at resolution 400 x 300 pixels with Photonfocus PHOT MV-D1024-160-CL camera). Visualization investigations aimed to identify nucleation sites and flow patterns and to determine the bubble departure diameter and frequency at various superheats for vertical tunnels. At low superheat vapor bubbles are generated nearly exclusively by the vertical tunnel. At medium values of superheat, pores of the horizontal tunnel activate.
\end{abstract}

\section{NOMENCLATURE}

d - diameter, $\mathrm{m}$

$f \quad$ - frequency, $\mathrm{Hz}$

$h \quad$ - height, $\mathrm{m}$

p - pitch, m

$s \quad$ - gap between fins, $\mathrm{m}$

w $\quad$ - width, $\mathrm{m}$

\section{Greek symbols}

$\Delta T \quad$ - superheat related to main fin base surface (=horizontal foil level), $\mathrm{K}$

$\delta \quad$ - thickness, $\mathrm{mm}$

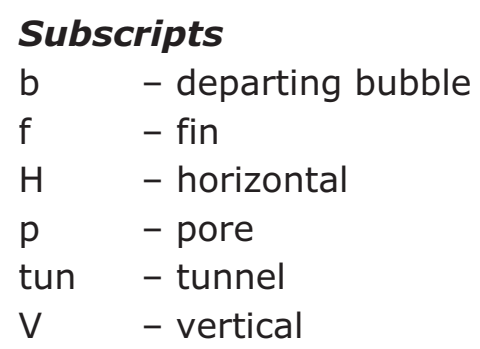

This is an Open Access article distributed under the terms of the Creative Commons Attribution License 2.0, which permits unrestricted use, distribution, and reproduction in any medium, provided the original work is properly cited. 


\section{INTRODUCTION}

Application of a complex surface combining advantages of a limited space, sub-surface tunnel and a porous layer (perforated foil or a mesh) is a new approach in the area of structures which enhance boiling heat transfer.

In order to formulate a theoretical model of boiling and for the choice of the proper structural surface geometry it is necessary to identify the sites of vapor bubble nucleation and to determine departing vapor bubble parameters.

The problem of external visualization on flat surfaces of enhanced structures has been mentioned in several publications over the past few years. Ramaswamy et al. [1], using a fast camera (1500 frames/s), determined the data concerning the bubble growth for a silicone structure composed of interconnected mini-channels, and placed in the boiling FC72. The data included the bubble departure diameter $(0.61-0.66 \mathrm{~mm})$ and frequency $(150$ $-250 \mathrm{~Hz}$ ).

Visualization for a similar structure, though made from copper and quartz and placed in PF 5060, was also performed by Ghiu and Joshi [2]. They showed different boiling regimes for the investigated materials and confirmed the dynamic nature of the boiling process.

$\mathrm{Yu}$ and Lu [3] presented boiling structure visualization results for copper mini-fins (the height: $0.5-4.0 \mathrm{~mm}$, the width of the inter-fin space: $0.5-2.0 \mathrm{~mm}$ ), immersed in the boiling FC-72 fluid at four different heat fluxes. The departing bubble diameters were estimated to be $0.2-0.3 \mathrm{~mm}$.

Also Pastuszko $[4,5]$ presented high speed external visualization of boiling heat transfer for the system of connected horizontal and vertical tunnels. The experiments were carried out with water at atmospheric pressure at heat fluxes of $130-290 \mathrm{~kW} / \mathrm{m}^{2}$. Diameter and frequency of departing bubbles were determined. Observations of internal and external visualizations confirmed most of the assumptions made for the semi-analytical model for boiling from enhanced structures composed of connected vertical and horizontal tunnels.

\section{EXPERIMENTAL SET-UP}

The visualization studies were conducted with a real sample (Fig. 1). The measurement stand for external visualization is a modified set-up described in [6].

The design of the module with rectangular glass vessel made it possible to observe the vapor bubble growth and bubble shape, to determine the size of the departing bubbles, and to determine the frequency at which vapor bubbles are generated at vertical tunnel outlets.

The diagram of external visualization stand is presented in Figure 1. Four transparent glass plates were attached to the edges of the sample base by means of epoxy adhesive. The plates were limiting the pool boiling volume and helped observe the boiling process from four directions. The whole module was sealed with high temperature silicone.

The visualization images were obtained using a high speed digital monochrome camera with CMOS sensor PHOT MV-D1024-160-CL (Photonfocus) of resolution 1024x1024 pixels at $150 \mathrm{fps}$. The visualization was created with the image acquisition speed of $493 \mathrm{fps}$ (at resolution $400 \times 300$ ). 


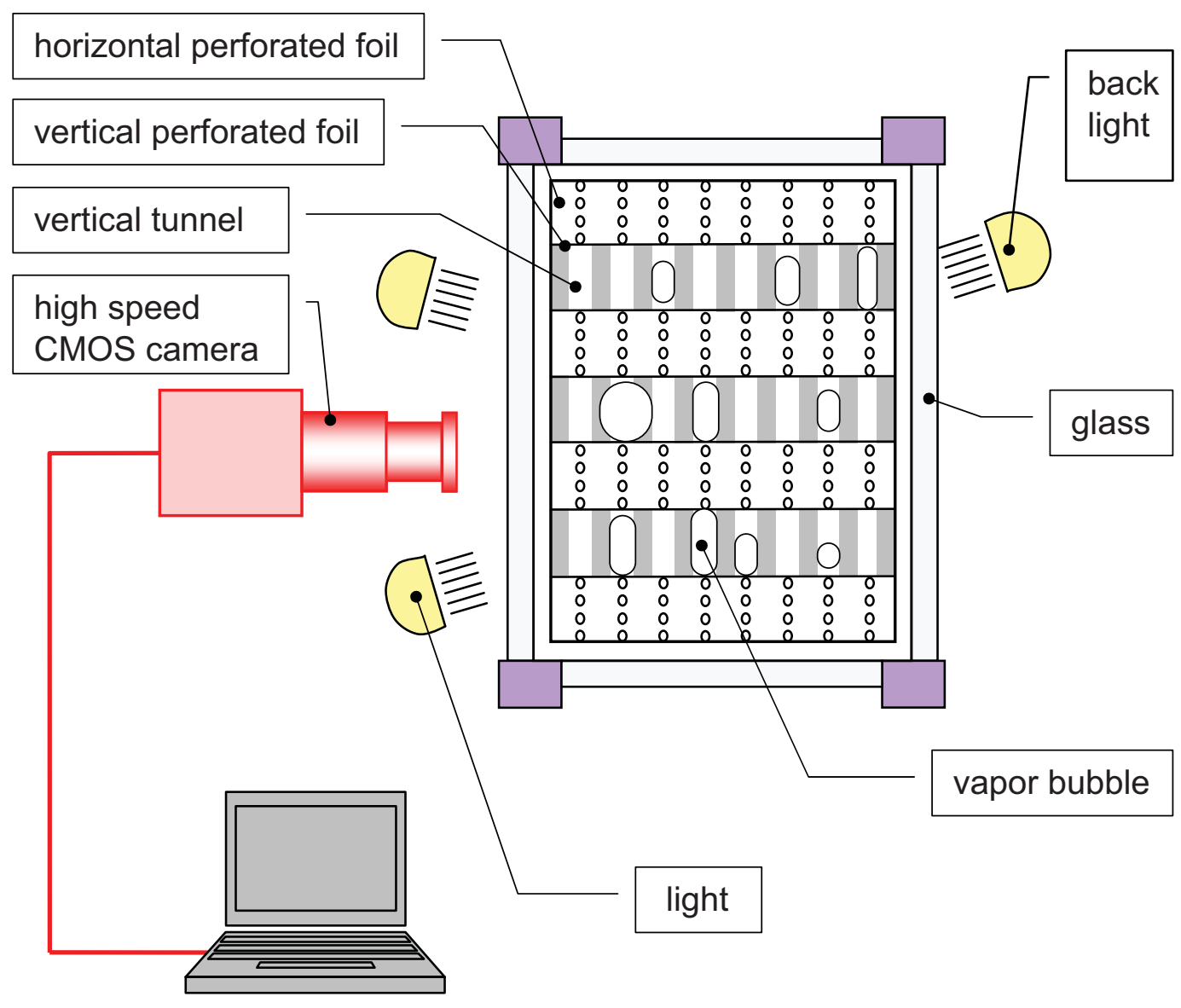

Figure 1. Schematic diagram of the visualization system.

Lighting was provided by a halogen lamp with light pipes (front light and back light). Temperature superheat $(\Delta T)$ was related to the level of the main fin base (it is also the level of the foil limiting horizontal tunnels). The quoted heat fluxes were also referred to the main fin base and it was determined from the temperature gradient in the upper part of the heating cylinder, assuming one-dimensional heat conduction.

The uncertainty in the wall superheat at main fin base, for $\Delta T=2-5 \mathrm{~K}$, was about \pm 0.2 $\mathrm{K}$.

\section{SAMPLE}

The surface for external visualization (Fig. 2) was composed of a system of 13 mini-fins arranged with $2 \mathrm{~mm}$ pitch ( $1 \mathrm{~mm}$ inter-fin space = tunnel width) and grouped in a form of three main fins. Perforated copper foil (holes diameter $0.3 \mathrm{~mm}$ ) was sintered on the lateral surfaces of the main fins and the tips of mini-fins of the horizontal inter-fin surface. The final result was a structure formed of joined narrow tunnels, both vertical and horizontal, with one wall made of perforated foil. 


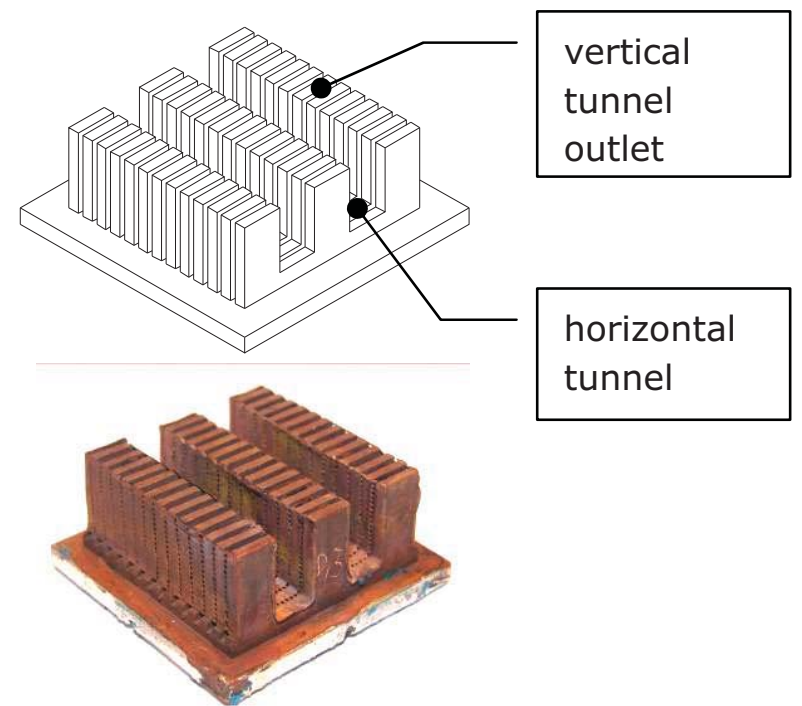

a)

Figure 2. Sample with narrow tunnel structure (NTS): a) view before porous foil sintering and a photo of a ready sample (NTS-10-1.0-0.3); b) vertical and horizontal section of the tunnel structure.

The parameters of the structure discussed above with the notations as in Figure 2 are shown in Table 1.

Table 1. Sample code and specifications.

\begin{tabular}{|l|l|l|l|l|l|l|l|l|l|}
\hline sample code & visualization type & $\begin{array}{l}d_{\mathrm{p},} \\
\mathrm{mm}\end{array}$ & $\begin{array}{l}p_{\mathrm{p},} \\
\mathrm{mm}\end{array}$ & $\begin{array}{l}h_{\mathrm{tunv},} \\
\mathrm{mm}\end{array}$ & $\begin{array}{l}h_{\mathrm{tunH},} \\
\mathrm{mm}\end{array}$ & $\begin{array}{l}w_{\text {tun }} \\
\mathrm{mm}\end{array}$ & $\begin{array}{l}p_{\text {tun },} \\
\mathrm{mm}\end{array}$ & $\begin{array}{l}\delta_{\mathrm{f}} \\
\mathrm{mm}\end{array}$ & $\begin{array}{l}s_{\prime} \\
\mathrm{mm}\end{array}$ \\
\hline NTS-10-1.0-0.3 & external & 0.3 & 0.6 & 10 & 1.0 & 1.0 & 2.0 & 5 & 5 \\
\hline
\end{tabular}

\section{Results}

\subsection{EXTERNAL VISUALIZATION}

Figures $3-5$ present photographs of the bubble growth phases in the vertical tunnel for heat fluxes $23-98 \mathrm{~kW} / \mathrm{m}^{2}$ (superheat $1.8-3.9 \mathrm{~K}$ ). The bubble, growing and departing at the vertical tunnel outlet, is marked with a circle.
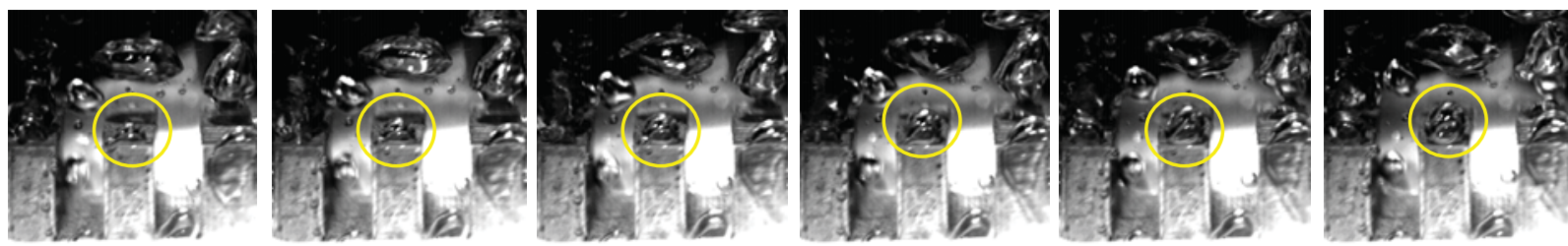

Figure 3. Bubble growth at the vertical tunnel outlet, boiling water: NTS-10-1.0$0.3, \Delta T=1.8 \mathrm{~K}, 493 \mathrm{fps}$. 

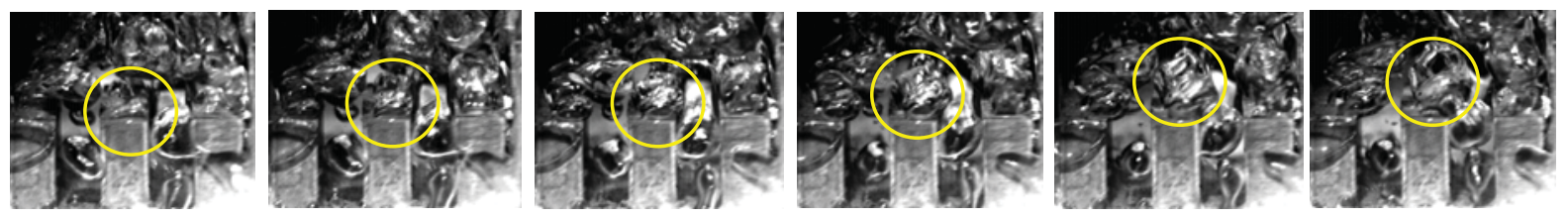

Figure 4. Bubble growth in the vertical tunnel NTS-10-1.0-0.3, $\Delta T=2.9 \mathrm{~K}, 493 \mathrm{fps}$ (every second frame).
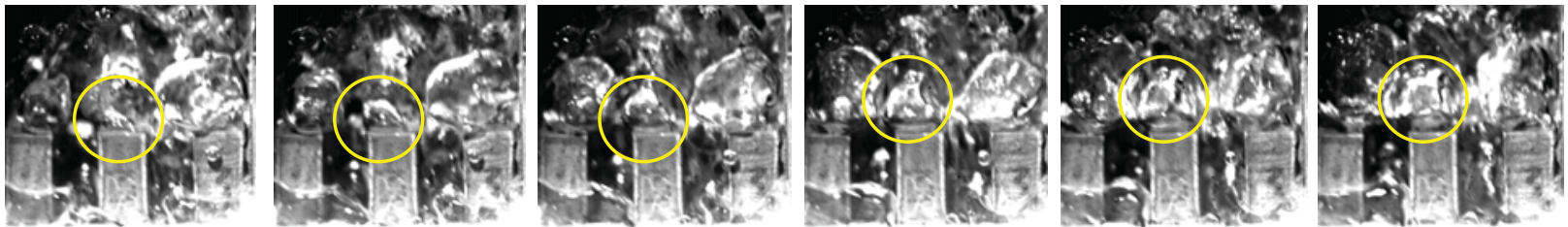

Figure 5. Bubble growth for the vertical tunnel NTS-10-1.0-0.3, $\Delta T=3.9 \mathrm{~K}, 493$ fps (every second frame).

At low superheats (Fig. 3) single vertical tunnel outlets are active in the main fins - initial boiling occurs only at these locations. The emerging bubble has a regular spherical shape; mean departure frequency at superheat reaching $1.8 \mathrm{~K}$ is about $24 \mathrm{~Hz}$. Horizontal tunnel pores are not active.

At the superheat of $2.9 \mathrm{~K}$, single bubbles growing from vertical tunnels and horizontal tunnel pores can be observed (Fig. 4). They usually have a shape of a flattened sphere. The diameter of the departing bubbles formed in vertical tunnels is $3.8-5.5 \mathrm{~mm}$ at the frequencies of about $32 \mathrm{~Hz}$.

When the superheat reaches 3.9 (Fig. 5) an extensive formation of vapor bubbles with irregular shapes is observed, and the bubbles which depart from the vertical tunnel outlets tend to merge within the area of the main fin. Large structures of mushroom-like shapes are formed, impeding the supply of liquid to horizontal tunnel pores. The departure frequency grows reaching $34 \mathrm{~Hz}$.

\subsection{BUBBLE DEPARTURE DIAMETER}

The values of the bubble mean diameter were calculated as the arithmetic average of the diameter of 6 bubbles measured in two directions (Fig. 6). The measurements were carried out for 5 superheats. Considering diameter measurement errors, the effect of the superheat changes is negligible for $\Delta T=1.8-3.9 \mathrm{~K}$. The average value of the bubble departure diameter in the investigated superheat range was $5 \mathrm{~mm}$, that is, three times the value of the vertical tunnel hydraulic diameter. The errors may be due to the fact that the tunnel width-length ratio was $1 / 5$ and some of the departing bubbles had a flattened shape. The flattening of the bubbles at higher superheats was also caused by the compact adherence of the bubbles departing from the tunnels distributed at the pitch of $2 \mathrm{~mm}$. 


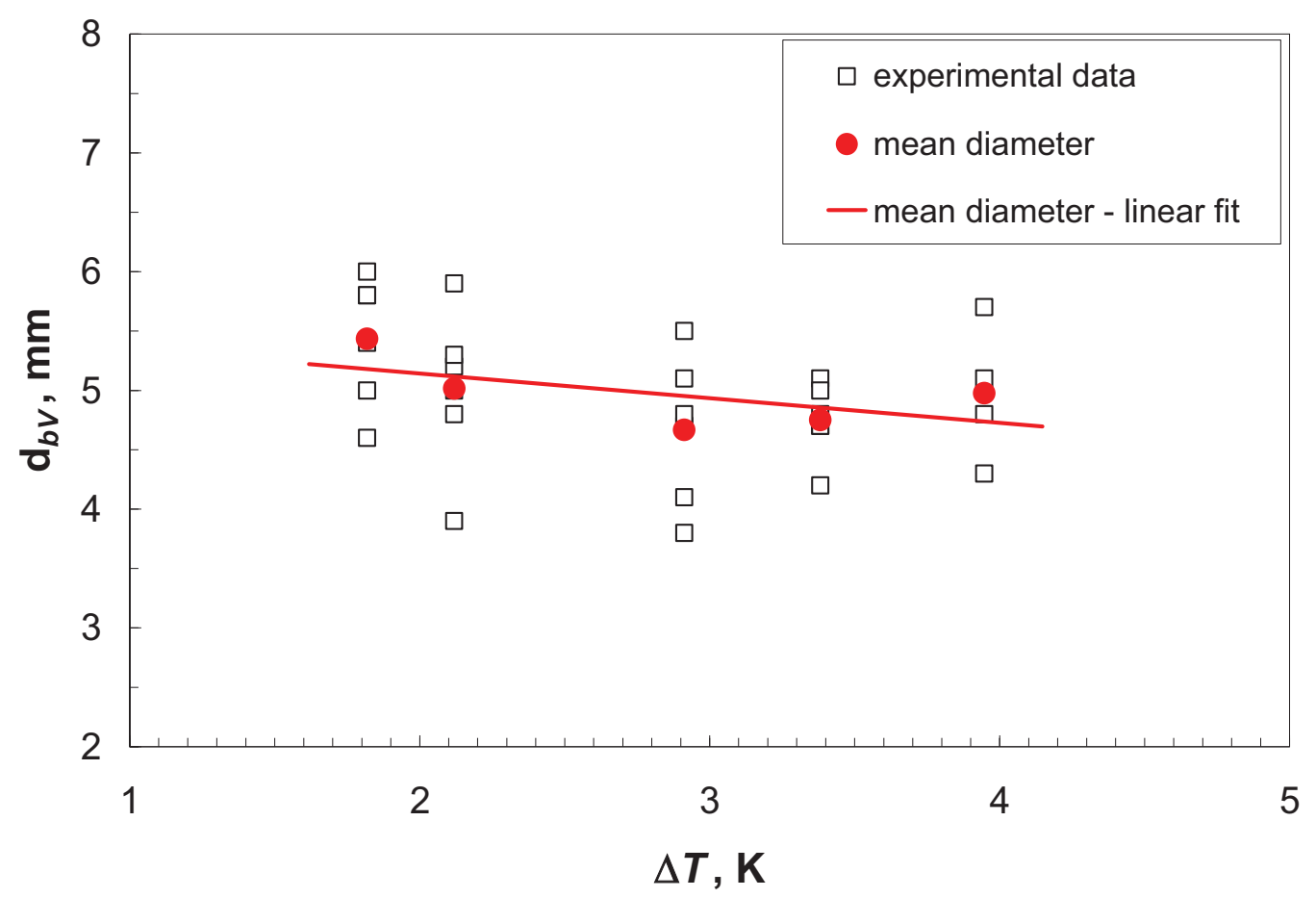

Figure 6. Bubble departure diameters at the outlets of vertical tunnels (for single, spherical bubbles).

Bubble diameters in each direction were computed based on pixel counts. The fastcamera picture resolution was $300 \times 400$ pixels. At the distance measurement error of \pm 2 pixels, the bubble diameter measurement error was about $\pm 0.2 \mathrm{~mm}$.

\subsection{BUBBLE DEPARTURE FREQUENCY}

At the vertical tunnel outlet, the bubble departure frequency increases insubstantially (Fig. 7) with the increasing superheat at main fin base $\Delta T$. For each superheat, frequencies determined for six randomly chosen tunnel outlets, were averaged. The average frequency of the departing bubbles for the selected tunnel outlet was calculated by determining the number of escaping bubbles on 100 recorded frames. Assuming that one bubble may be added or omitted by mistake when counting bubbles departing from one tunnel outlet at $0.474 \mathrm{~s}$ (100 frames), the frequency measurement error is $\pm 2 \mathrm{~Hz}$.

The graph and the visualization frames show that at low superheats only a small number of bubbles depart from vertical tunnel outlets. This phenomenon may be the result of a substantial temperature drop at the height of the main fin at low values of $\Delta T$ - the local superheat in the upper parts of the fin, that is at the vertical tunnel outlet level, would be insufficient to initiate bubble boiling. 


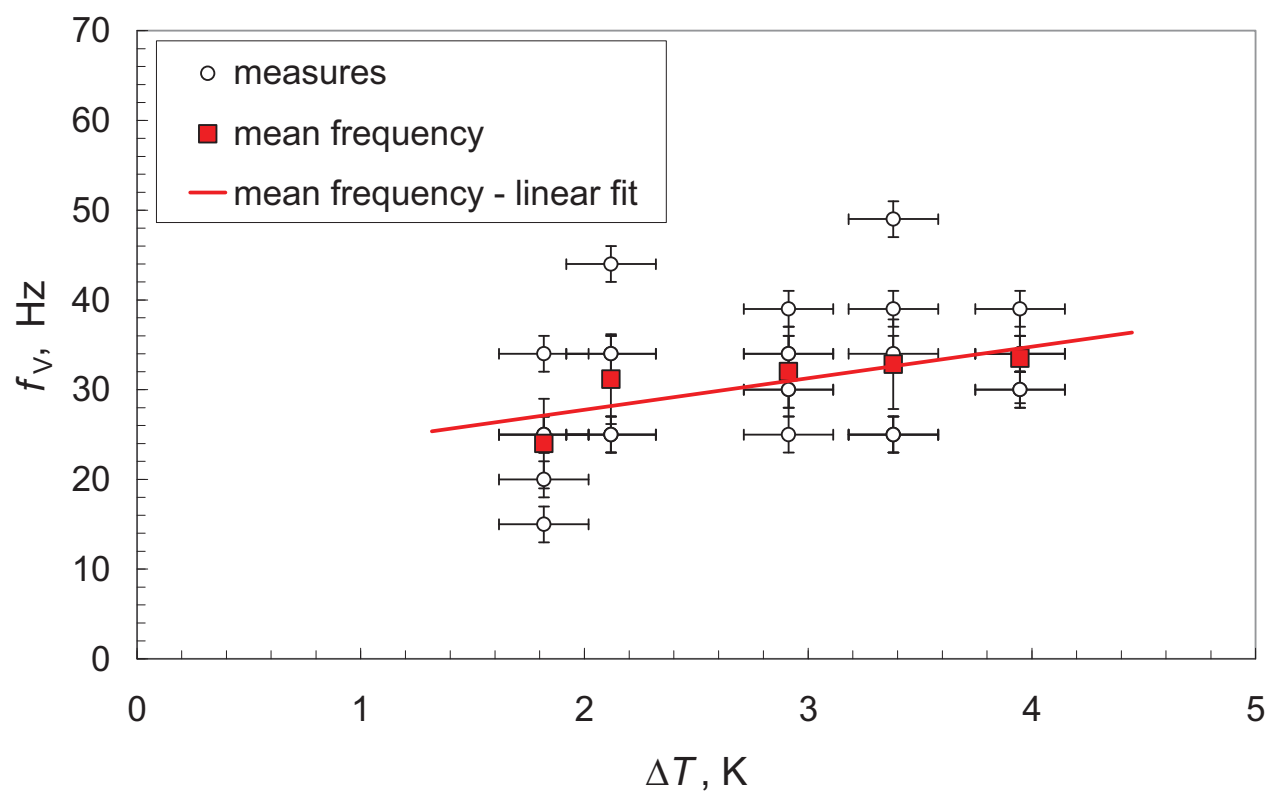

Figure 7. Bubble departure frequencies at the outlets of vertical tunnels.

\section{Conclusions}

The visualization experiments allow drawing the following conclusions:

- Pores in the perforated foil of vertical tunnel are inactive - their task it only to provide subsurface tunnels with liquid. Liquid layer evaporation in the vertical tunnel is connected with a fast upward movement of the bubbles toward the outlet.

- Vertical tunnel outlets are active within the whole range of investigated heat fluxes.

- In the superheat range $1.8-3.9 \mathrm{~K}$ the diameters of departing bubbles are $5 \pm 1 \mathrm{~mm}$, and the mean diameter is $5 \pm 0.4 \mathrm{~mm}$.

- In the mentioned superheat range, the mean frequency of the departing bubbles increases from 24 to $34 \mathrm{~Hz}$.

\section{REFERENCES}

[1] Ramaswamy C., Joshi Y., Nakayama W., Johnson W. B.: High-speed visualization of boiling from an enhanced structure, Int. J. Heat Mass Transfer, 45 (2002), 4761-4771.

[2] Ghiu C.-D., Joshi Y.K.: Visualisation study of pool boiling from thin confined enhanced structures, Int. J. Heat Mass Transfer, 48 (2005), 4287-4299.

[3] Yu C. K., Lu D. C.: Pool boiling heat transfer on horizontal rectangular fin array in saturated FC-72, Int. J. Heat Mass Transfer, 50 (2007), 3624-3637.

[4] Pastuszko R.: Pool boiling visualization for surfaces with narrow tunnels limited by porous structure, proc. 5th European Thermal-Sciences Conference, 18-22 May 2008, Eindhoven, the Netherlands, TPF_23.

[5] Pastuszko R.: High speed boiling visualization for fins with subsurface tunnels, proc. 7th World Conference on Experimental Heat Transfer, Fluid Mechanics and Thermodynamics (ExHFT-7), Krakow, Poland, June 28 - July 03, 2009, HT-39.

[6] Pastuszko R.: Boiling heat transfer enhancement in subsurface horizontal and vertical tunnels, Experimental Thermal and Fluid Science, 32 (2008), 15641577. 